\title{
Cadaveric comparison of the accuracy of ultrasound-guided versus 'blind' perineural injection of the tibial nerve in horses
}

\author{
Maylin van der Laan ${ }^{\mathrm{a}, \mathrm{b}, *}$, Els Raes ${ }^{\mathrm{a}}$, Maarten Oosterlinck ${ }^{\mathrm{b}}$ \\ a Department of Veterinary Medical Imaging and Small Animal Orthopedics, Faculty of Veterinary Medicine, Ghent University, Salisburylaan 133, 9820 \\ Merelbeke, Belgium \\ ${ }^{\mathrm{b}}$ Department of Surgery and Anaesthesiology of Domestic Animals, Faculty of Veterinary Medicine, Ghent University, Salisburylaan 133, 9820, Merelbeke, \\ Belgium
}

\section{A R T I C L E I N F O}

\section{Article history:}

Accepted 21 December 2020

\section{Keywords:}

Diagnostic anaesthesia

Nerve block

Lameness examination

Orthopaedics

Regional anaesthesia

\begin{abstract}
A B S T R A C T
During diagnostic evaluation of hindlimb lameness in horses the tibial nerve block is traditionally performed based on anatomical reference points, but it can be difficult to achieve effective local anaesthesia using this blind technique. Ultrasound (US)-guided injection could increase the accuracy of injection. The aim of this study was to compare the accuracy of both techniques. Twenty-one paired sets of cadaver hindlimbs were injected with $1 \mathrm{~mL}$ methylene blue using the blind or US-guided technique. There was no significant difference in stain width and length and in coloured nerve length between techniques. However, the successful rate of nerve staining was $85.7 \%$ and $47.6 \%$ for the US-guided and blind technique, respectively $(P=0.02$; odds ratio 6.6 ; $95 \%$ confidence interval, $1.5-29.4)$. This study suggests that the US-guided technique is more accurate than the blind technique. However, in the treated sample, a single US-guided injection did not consistently result in nerve staining.
\end{abstract}

(c) 2021 Elsevier Ltd. All rights reserved.
Nerve blocks are often used in the diagnostic evaluation of lameness in horses. A tibial nerve block can be used when more distal nerve and/or intra-articular blocks have not resulted in improvement of the hindlimb lameness (Bassage and Ross, 2011). The tibial nerve emerges between the medial head of the gastrocnemius muscle and the superficial digital flexor muscle on the medial side of the crus. The nerve runs cranial to the common calcaneal tendon and is contained within the superficial caudal crural compartment limited by the superficial and deep caudal crural fasciae (Budras et al., 2012; Denoix et al., 2020).

The blind technique consists of injecting $15-20 \mathrm{~mL}$ of local anaesthetic solution with a $20-22 \mathrm{G}, 2.5 \mathrm{~cm}$ needle in the lateral or medial side of the limb, $10 \mathrm{~cm}$ proximal to the tuber calcanei and 1 $\mathrm{cm}$ cranial to the common calcaneal tendon (Wheat and Jones, 1981; Dyson, 1984; Skarda et al., 2009; Bassage and Ross, 2011). Based on the size of the tibial nerve, it has been recommended to wait 20-30 min before evaluating the result (Bassage and Ross, 2011). However, the authors' practical experience suggests that it can be difficult to achieve effective local anaesthesia, even after substantial waiting time and even using larger volumes of local anaesthetic solution.

\footnotetext{
* Corresponding author at: Horse practice de Waert, Oud-heinenoordseweg 18, 3274 KD Heinenoord, The Netherlands.

E-mail address: maylin@live.nl (M. van der Laan).
}

Ultrasound (US)-guided injection could increase the accuracy of injection, as stated by Denoix et al. (2020) albeit without direct comparison between blind and US-guided techniques. Ultrasoundguided injection allows deposition of local anaesthetic solution as close as possible to the nerve and especially within the superficial caudal crural compartment (Denoix et al., 2020). A detailed description of the US-guided technique is provided in the Appendix (Supplementary Item 1). The aim of this study was to compare the accuracy of the conventional blind technique and the US-guided technique for perineural injection of the tibial nerve with methylene blue in cadaveric limbs of horses. It was hypothesised that the US-guided technique would result in superior accuracy compared to the blind technique. Therefore, an ex vivo study was performed on 42 cadaver hindlimbs from 21 horses euthanised at the Faculty of Veterinary Medicine of Ghent University for various reasons unrelated to hindlimb pathology. Research ethics committee oversight was not required as the study was performed using material collected during post-mortem examinations. Sex, age and breed of the horses included in this study were not recorded. Hindlimbs were disarticulated at the coxofemoral joint and stored in a refrigerator $\left(4^{\circ} \mathrm{C}\right)$ in batches of two to three horses. All limbs were tested within three days of disarticulation. Paired hindlimbs were randomly assigned to be injected using either the blind or US-guided technique, using simple randomisation by a random number list generated in spreadsheet software (Microsoft Excel). The limbs were placed 
with the lateral side facing down and the limb in semi-extension; the region of interest was clipped prior to injection. Injection was performed $20 \mathrm{~min}$ after equilibration of the limb to room temperature.

For the blind technique, $1 \mathrm{~mL}$ methylene blue was injected through a $21 \mathrm{G}, 2.5 \mathrm{~cm}$ hypodermic needle which was inserted approximately $1 \mathrm{~cm}$ deep, at the medial side of the limb, $10 \mathrm{~cm}$ proximal to the tuber calcanei and $1 \mathrm{~cm}$ cranial to the common calcaneal tendon (Skarda et al., 2009; Fig. 1). This technique was performed by a board-certified (ECVS, ECVSMR) specialist operator (MO) with extensive clinical experience of this procedure. For the US-guided technique, the region of interest was washed and subsequently rinsed with $70 \%$ denatured ethanol. After ultrasonographic visualisation of the tibial nerve (Fig. 2) using a linear probe at frequency of $7.5 \mathrm{MHz}$ (Philips CX 50), a 21G, $2.5 \mathrm{~cm}$ needle was inserted cranial to the nerve under ultrasound guidance and $1 \mathrm{~mL}$ methylene blue was injected. The US-guided technique was performed by a board-certified (ECVDI) specialist operator (ER) with expertise in ultrasound-guided procedures.

Five min after performing the injection, the skin medial and proximal to the tarsus was removed to measure the length and width of the methylene blue stain (Fig. 3). Subsequently, the tibial nerve was further dissected, and it was determined whether the nerve was coloured or not. If the nerve was coloured, the length of the nerve coloured by methylene blue was measured (Fig. 4). If the nerve was not coloured, the coloured anatomical structure (e.g. superficial caudal crural fascia) was further dissected and measured (maximum distribution, i.e. largest width (cranio-caudal direction) and length (proximo-distal direction) of the stain). Moreover, it was determined if the stain was caudal, cranial, medial or lateral to the nerve and the distance between the edge of the stain and the nerve was measured. A ruler was used for all length

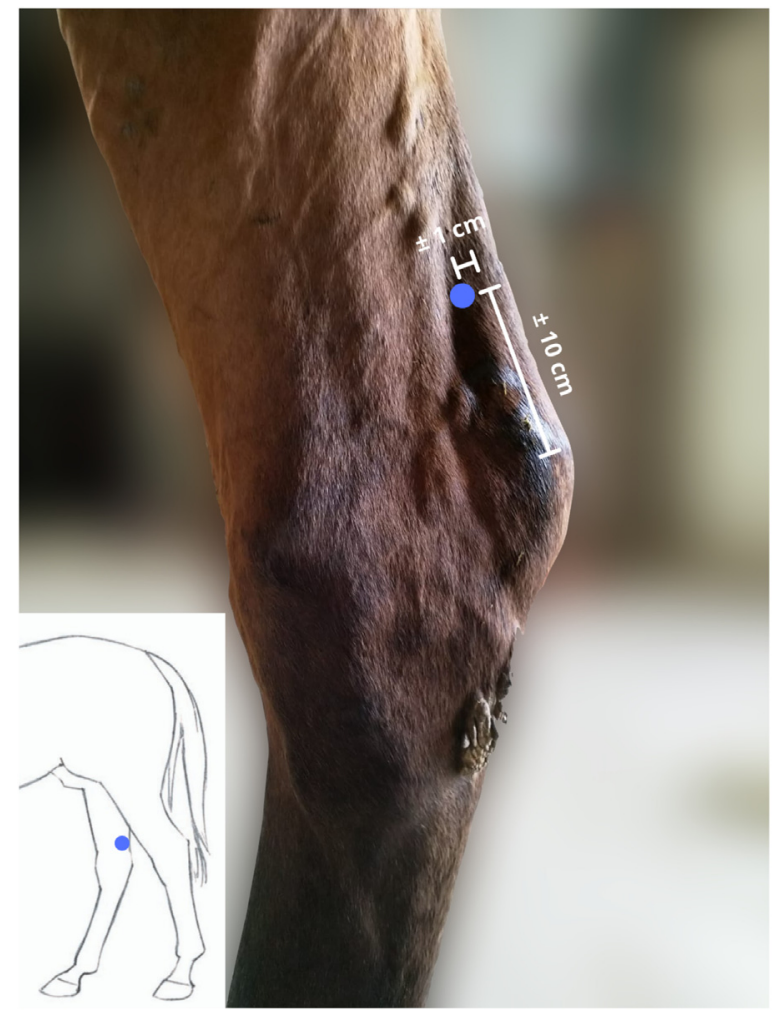

Fig. 1. Illustration of the injection site (blue dot) at the medial side of the hindlimb, $10 \mathrm{~cm}$ proximal to the tuber calcanei and $1 \mathrm{~cm}$ cranial to the common calcaneal tendon.

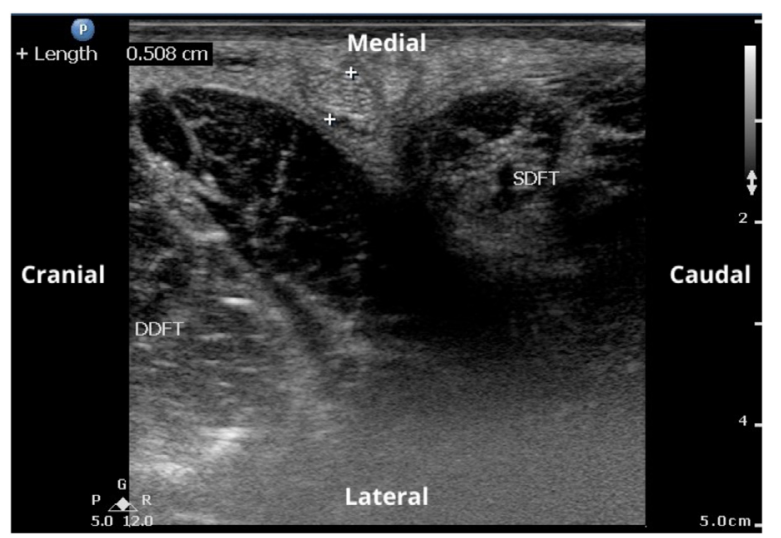

Fig. 2. Transverse ultrasound section of the caudomedial part of an equine crus (+, tibial nerve; SDFT, superficial digital flexor tendon; DDFT, deep digital flexor tendon).

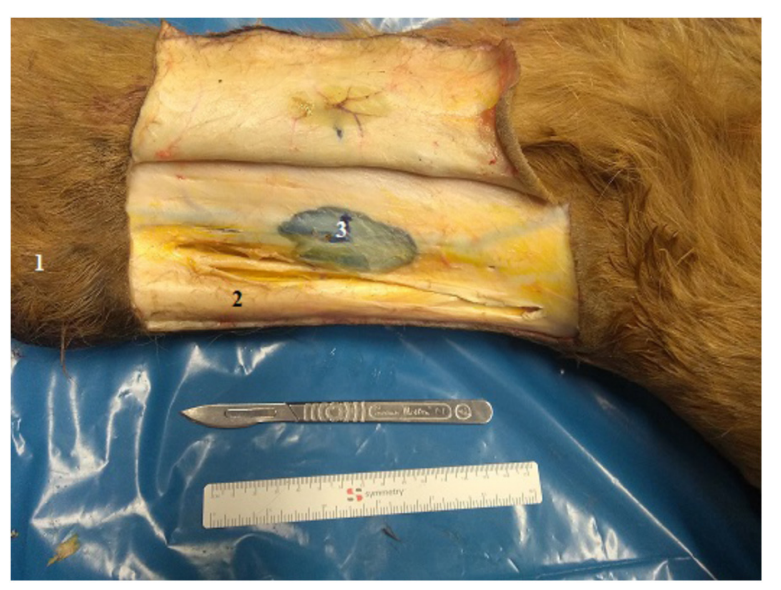

Fig. 3. Example of the medial aspect of an equine crus after removing the skin (right is proximal; (1) tuber calcanei; (2) common calcaneal tendon; (3) methylene blue stain).

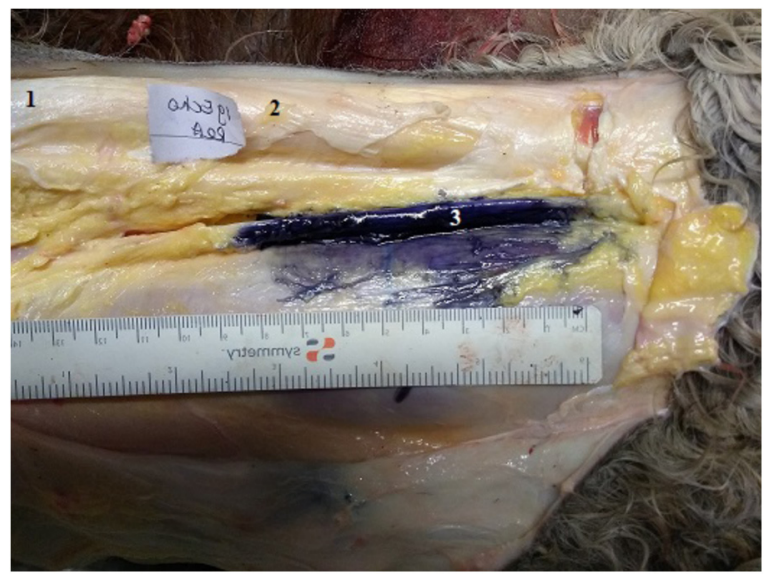

Fig. 4. Example of the medial aspect of an equine crus after removing the skin and dissecting the tibial nerve (right is proximal; (1) tuber calcanei; (2) common calcaneal tendon; (3) tibial nerve).

measurements. The researcher performing the dissections was not blinded to the injection technique.

Data were tested for normality using Kolmogorov-Smirnov and Shapiro Wilk test, and Wilcoxon signed rank test (methylene blue 
stain width), paired Student's $t$-test (methylene blue stain length) and Mann-Whitney $U$ test (coloured nerve length) were used to compare data between the US-guided and blind technique. Fisher's exact test was used to test for association between the technique used and nerve staining results, including calculation of the odds ratio and $95 \%$ confidence interval $(95 \% \mathrm{CI})$. All statistical tests were performed with SPSS Statistics 25 (IBM), with statistical significance set at $P<0.05$. Data are presented as mean \pm standard deviation (SD) or median, interquartile range.

There was no significant difference in the stain length $(P=0.16)$ between the blind $(6.18 \pm 2.07 \mathrm{~cm})$ and the US-guided technique $(7.04 \pm 1.61 \mathrm{~cm})$. There was also no significant difference in stain width $(P=0.54)$ between the blind $(1.5 \mathrm{~cm}, 1.2)$ and the US-guided technique $(1.5 \mathrm{~cm}, 0.75)$. No significant difference was found in the length of the coloured nerves $(P=0.13)$ between the blind $(4.5 \mathrm{~cm}$, 6.4) and the US-guided technique $(6.75 \mathrm{~cm}, 4.5)$. However, injections resulting in coloured nerves were significantly more often observed with the US-guided technique (85.7\%) than with the blind technique ( $47.6 \% ; P=0.02$; OR $6.6 ; 95 \% \mathrm{CI}, 1.5-29.4$ ). In the cases without nerve staining, the distance between the nerve and the methylene blue stain was $0.1 \mathrm{~cm}$ in the US-guided group (in all cases) and $0.25 \pm 0.34 \mathrm{~cm}$ in the group using the blind technique. This could not be compared statistically because of the low number of non-coloured nerves in the US-guided group $(n=3 / 21)$ compared with the blind group $(n=11 / 21)$. Dye deposition did not indicate any potential risks associated with either technique (e.g. intrasynovial deposition).

Our results confirm that when the tibial nerve is approached in an isolated limb positioned on a table generating optimal access conditions, using the US-guided technique produces a more predictable result than not using it, based on the significantly higher proportion of coloured nerves with the US-guided technique. The odds ratio showed an important difference but a low precision (95\% CI, 1.5-29.4), which is a consequence of the small sample size. In the three cases in which the nerve was not coloured after the US-guided technique, the methylene blue was just $1 \mathrm{~mm}$ distant to the nerve when measured at dissection, under the deep crural fascia (Fig. 5) which precluded distribution of the methylene blue. A similar observation was made for most cases in which the nerve was not coloured after the blind technique, but interestingly, in two of those cases, the methylene blue was injected in the perineural fat but without reaching the nerve (Fig. 6). Although the sample size was too small to allow statistical comparison of this variable between groups, it illustrates the

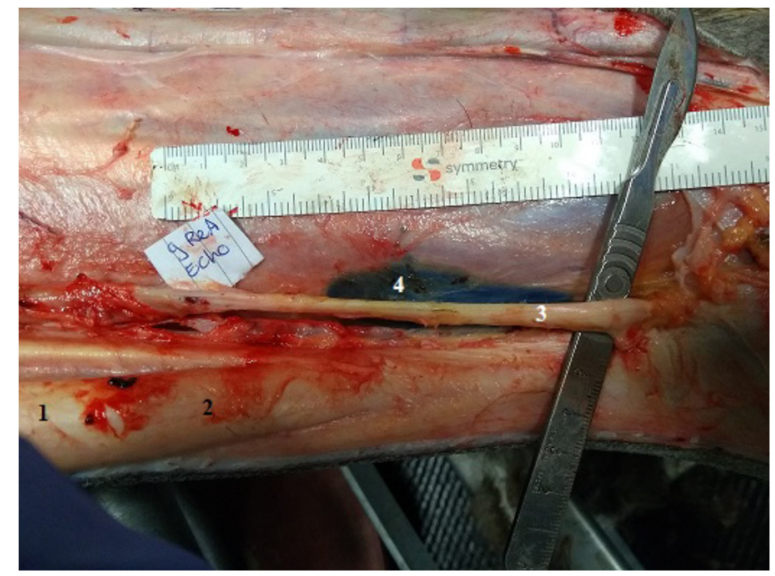

Fig. 5. Example of a specimen in which the nerve was not coloured after the USguided technique, with the methylene blue injected $1 \mathrm{~mm}$ too deep, under the deep crural fascia which precluded diffusion of the methylene blue (right is proximal; (1) tuber calcanei; (2) common calcaneal tendon; (3) tibial nerve; (4) methylene blue stain). possible contribution of the fascia and perineural fat in inhibition of the distribution of the methylene blue.

Performing the US-guided technique safely on a live horse without sedation can be challenging, because of the additional equipment and personnel required as recently described by Denoix et al. (2020). In that report, it was recommended to have two people restraining the horse and two operators, each positioned on one side of the injected leg. Denoix et al. (2020) used a $1.5 \mathrm{~cm} 25 \mathrm{G}$ hypodermic needle and a $6-10 \mathrm{MHz}$ micro convex probe to inject 5-8 $\mathrm{mL}$ of local anaesthetic solution both cranial and caudal to the tibial nerve, whereas in the present study, we only injected cranially and used a very small volume of methylene blue $(1 \mathrm{~mL})$ to assess accuracy of injection. With the increased accuracy of the USguided technique, a smaller volume of anaesthetic solution may be sufficient for obtaining a successful block of the tibial nerve, although this requires further investigation in vivo.

Two different operators (one specialist clinician experienced in using the blind technique and one experienced in US-guided injections) were used for the two techniques, to avoid that operator preference or experience with one technique would create bias in the results. Theoretically, it would have been preferable to have one single operator with no preference and equal experience in blind and US-guided injection techniques, but this was not considered achievable for the present study.

In the present study, a linear probe with a frequency of $7.5 \mathrm{MHz}$ was used, which allowed sufficient detail to visualise the nerve on these cadaver limbs. However, a micro convex probe may be easier to handle on a live horse. Moreover, a higher frequency would have resulted in a better resolution and in combination with the depth of the nerve to be stained, this may have affected the success of the US-guided technique. It is therefore recommended to optimize visualisation of the superficial and deep caudal crural fasciae to ensure correct injection. Regarding the needle length, the rather superficial depth of the tibial nerve did not require full insertion of the $2.5 \mathrm{~cm}$ needle in the blind technique, however in the US-guided technique, entry angle and probe width must also be considered and a $1.5 \mathrm{~cm}$ needle as used by Denoix et al. (2020) might be insufficient when using a linear probe.

In conclusion, this study confirms that under optimal access conditions, the US-guided technique for the tibial nerve block may be more accurate than the blind technique. However, even under ultrasound guidance, a single injection did not consistently result in nerve staining due to puncturing the deep caudal crural fascia. This may be overcome by using a higher transducer frequency and/

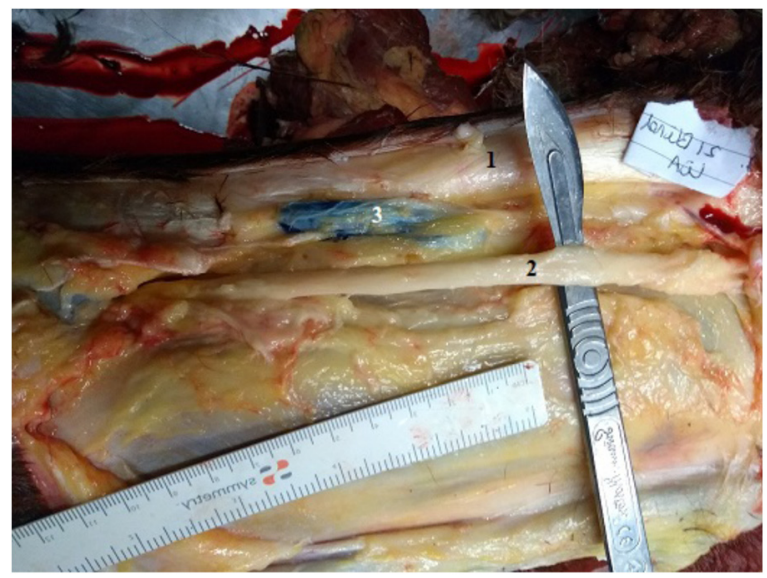

Fig. 6. Example of a specimen in which the nerve was not coloured after the blind technique; with the methylene blue injected in the perineural fat but without reaching the nerve (right is proximal; (1) common calcaneal tendon; (2) tibial nerve; (3) methylene blue stain). 
or by injecting cranial and caudal to the nerve as described by Denoix et al. (2020).

\section{Conflict of interest}

None declared.

\section{Acknowledgements}

We thank the technical staff of the Department of Pathology, Bacteriology and Poultry Diseases of the Faculty of Veterinary Medicine of Ghent University of their help with collecting the specimens.

\section{Appendix A. Supplementary data}

Supplementary material related to this article can be found, in the online version, at doi:https://doi.org/10.1016/j.tvjl.2020.105603.

\section{References}

Bassage, L.H., Ross, M.W., 2011. Diagnostic analgesia, In: Ross, M.W., Dyson, S.J. (Eds.), Diagnosis and Management of Lameness in the Horse. Second ed. Saunders Elsevier, St. Louis, MO, USA, pp. 100-135.

Budras, K., Berg, R., Horowitz, A., Rock, S., Sack, W.O., 2012. Pelvic limb, In: Budras, K. Sack, W.O. (Eds.), Anatomy of the Horse. Sixth ed. Schleutersche, Hannover, Germany, pp. 130-141.

Denoix, J.-M., Beaumont, A., Bertoni, L., 2020. Ultrasonographic guided block of the tibial nerve. Equine Veterinary Education 32, 372-377.

Dyson, S., 1984. Equine practice nerve blocks and lameness diagnosis in the horse. In Practice 6, 102-107.

Skarda, R.T., Muir, W.W., Hubbell, J.A.E., 2009. Local anaesthetic drugs and techniques, In: Muir, W.W., Hubbell, J.A.E. (Eds.), Equine Anaesthesia. Second ed. Saunders Elsevier, St. Louis, MO, USA, pp. 210-242.

Wheat, J.D., Jones, K., 1981. Selected techniques of regional anaesthesia. Veterinary Clinics of North America: Large Animal Practice 3, 223-246. 